\title{
MTF Measurement Based on Interactive Live-Wire Edge Extraction
}

\author{
Peng Liu ${ }^{1,2,3}$, Dingsheng $\mathrm{Liu}^{1}$, and Fang Huang ${ }^{1,3}$ \\ ${ }^{1}$ China Remote Sensing Satellite Ground Station, \#45, Bei San Huan Xi Road, Beijing, China \\ ${ }^{2}$ Institute of electronic Chinese academy of science, \#19, Bei Si Huan Xi Road, Beijing, China \\ \{pliu, dsliu, thuang\}@ne.rsgs.ac.cn \\ ${ }^{3}$ Graduate University of Chinese Academy of Sciences
}

\begin{abstract}
When we want to measure parameters of the Modulation Transfer Function (MTF) from remote sensing image directly, the sharp edges are usually used as targets. But for noise, blur and the complexity of the images, fully automatic locating the expected edge is still an unsolved problem. This paper improves the semi-auto edge extraction algorithm of live-wire [1] and introduces it into the knife-edge method [8] of MTF measuring in remote sensing image. Live-wire segmentation is a novel interactive algorithm for efficient, accurate, and reproducible boundary extraction that requires minimal user input with a mouse. The image is transformed into a weighted graph with variety restrictions. Edge searching is based on dynamic programming of Dijkstra's algorithm [5]. Optimal boundaries are computed and selected at interactive rates as the user moves the mouse starting from a manually specified seed point. In this paper, a promoted model of live-wire is proposed to measuring the on orbit Modulation Transfer Function for high spatial resolution imaging satellites. We add the no-linear diffusion filter in the local cost function to ensure the accurateness of the extraction of edges. It can both de-noise and do not affect the shape of the edges when we extracting the edges, so that the calculation of the MTF is more reasonable and precise.
\end{abstract}

Keywords: MTF measurement, interactive Live-wire edge extraction, sharp edge.

\section{Introduction}

In order to measure the on orbit MTF of remote sensing images, knife-edges method makes use of special targets for evaluating spatial response since the targets stimulate the imaging system at all spatial frequencies [8]. The algorithm must determine edge locations with very high accuracy, such as Figure 1.1(a). The ESF (edge spread function) was then differentiated to obtain the LSF (line spread function) as in the second picture in Figure 1.1(d). Then the LSF was Fourier-transformed and normalized to obtain the corresponding MTF, see Figure 1.1 (e).

So like Figure1.2 (a) and (b), we hope that we can extract the edges arbitrarily and like figure1.2 (c), we can acquire the perpendicular profiles easily. But for the 
complexity of the images, fully automated locating the expected edge is still an unsolved problem. Especially, in most case the edge in the image could be not straight or regular. So the aim to cut a profile that is perpendicular to the edge is also very difficult. As far as above reasons are considered, we introduce "live-wire" [1] into MTF measurement and promote the novel method of edges detecting. Live-wire is one of active contour [2] model for efficient, accurate boundary extraction. Optimal boundaries are computed and selected at interactive rates as the user moves the mouse starting from a manually specified seed point. In this paper we enhance the performance of the live-wire model and make it more fitful to MTF measurement in the remote sensing images.

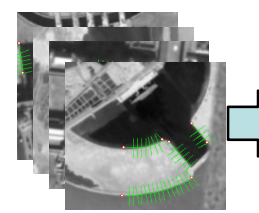

(a)

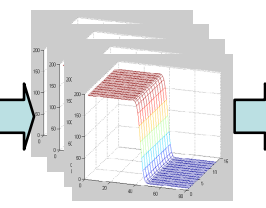

(b)

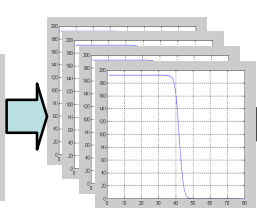

(c)

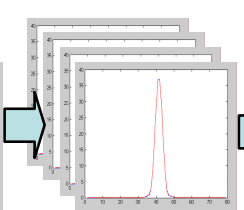

(d)

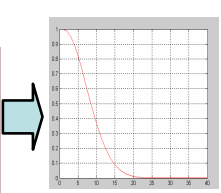

(e)

Fig. 1.1. The process from the sharp edges to MTF

\section{Live-Wire Model}

The motivation behind the live-wire algorithm is to provide the user with full control over the edge extraction [2]. Initially, the user clicks to indicate a starting point on the desired contour, and then as the mouse is moved it pulls a "live-wire" behind it along the contour. When the user clicks again, the old wire freezes on the contour, and a new live one starts from the clicked point. The livewire method poses this problem as a search for shortest paths on a weighted graph. Thus the livewire algorithm has two main parts: first, the conversion of image information into a weighted graph, and then the calculation of shortest paths in the graph [6],[9]. This paper for the first time introduces the algorithm into MTF measurement and makes some good promotions.

In the first part of the livewire algorithm, weights are calculated for each edge in the weighted graph, creating the image forces that attract the livewire. To produce the weights, various image features are computed in a neighborhood around each graph edge, and then combined in some user-adjustable fashion. The general purpose of the combination is edge localization, but individual features are generally chosen as in [9]. First, features such as the gradient and the Laplacian zero-crossing [3] have been used for edge detection. Second, directionality, or the direction of the path should be taken consideration locally. Third, training is the process by which the user indicates a preference for a certain type of boundary. For resisting the affections of noise, this paper makes promotion by adding the no-linear diffusion filter term, which we can see in the following contents. 
In the second part of the livewire algorithm, Dijkstra's algorithm [5] is used to find all shortest paths extending outward from the starting point in the weighted graph. The livewire is defined as the shortest path that connects two user-selected points (the last clicked point and the current mouse location). This second step is done interactively so the user may view and judge potential paths, and control the extraction as finely as desired. And this will make MTF measurement easier.

\subsection{Weighted Map and Local Cost}

The local costs are computed as a weighted sum of these component functions, such as Laplacian zero-Crossing, Gradient Magnitude and Gradient Direction. Letting $l(p, q)$ represents the local cost on the directed link from pixel $p$ to a neighboring pixel $q$, the local cost function is

$$
l(p, q)=w_{Z} \cdot f_{Z}(q)+w_{D} \cdot f_{D}(p, q)+w_{G} \cdot f_{G}(q)+w_{d i v} f_{d i v}(p)
$$

In formula (1), $f_{\text {div }}(p)$ is Laplacian zero-Crossing, $f_{G}(q)$ is Gradient Magnitude, $f_{D}(p, q)$ is Gradient Direction and $f_{\text {div }}(p)$ is Divergence of Unit Gradient. $w_{Z}$, $w_{D}, w_{G}$ and $w_{\text {div }}$ are their weights, and each $w$ is the weight of the corresponding feature function. The Laplacian zero-crossing is a binary edge feature used for edge localization [7]. The Laplacian image zero-crossing corresponds to points of maximal (or minimal) gradient magnitude. Thus, Laplacian zero-Crossings represent "good" edge properties and should therefore have a low local cost. If $I_{L}(q)$ is the Laplacian of an image $I$ at the pixel $q$, then

$$
f_{Z}(q)=\left\{\begin{array}{lll}
0 & \text { if } & I_{L}(q)=0 \\
1 & \text { if } & I_{L}(q) \neq 0
\end{array}\right.
$$

Since the Laplacian zero-crossing creates a binary feature, $f_{Z}(q)$ does not distinguish between strong, high gradient edges and weak, low gradient edges. However, gradient magnitude provides a direct correlation between edge strength and local cost. If $I_{x}$ and $I_{y}$ represent the partials of an image $I$ in $x$ and $y$ respectively, then the gradient magnitude $G$ is approximated with $G=\sqrt{I_{x}^{2}+I_{y}^{2}}$. Thus, the gradient component function is

$$
f_{G}=\frac{\max (G-\min (G))-(G-\min (G))}{\max (G-\min (G))}=1-\frac{G-\min (G)}{\max (G-\min (G))}
$$

The gradient direction $f_{D}(p, q)$ adds a smoothness constraint to the boundary by associating a high cost for sharp changes in boundary direction. The gradient direction is the unit vector defined by $I_{x}$ and $I_{y}$. Letting $D(p)$ be the unit vector perpendicular 
to the gradient direction at the point $p$ (for $D(p)=\left(I_{y}(p),-I_{x}(p)\right)$ ), the formulation of the gradient direction feature cost is:

$$
\begin{aligned}
& f_{D}(p, q)=\frac{1}{\pi}\left\{\cos [D(p) \bullet L(p, q)]^{-1}+\cos [L(p, q) \bullet D(p)]^{-1}\right\} \\
& \left\{\begin{array}{l}
D(p)=\left(I_{y}(p)-I_{x}(p)\right) \\
D(q)=\left(I_{y}(q)-I_{x}(q)\right)
\end{array}\right. \\
& L(p, q)=\frac{1}{\|p-q\|}\left\{\begin{array}{lll}
q-p ; & \text { if } & D(p) \bullet(q-p) \geq 0 \\
p-q ; & \text { if } & D(p) \bullet(q-p) \prec 0
\end{array}\right. \\
& f_{d i v}(p)=-\left(\frac{\partial}{\partial x}\left(\frac{I_{x}}{\sqrt{I^{2}{ }_{x}+I^{2}{ }_{y}+\beta}}\right)+\frac{\partial}{\partial y}\left(\frac{I_{y}}{\sqrt{I^{2}{ }_{x}+I^{2}{ }_{y}+\beta}}\right)\right)
\end{aligned}
$$

Above, in $D(p) \bullet L(p, q)$ of (4), “•” is vector dot products. And here, (5) and (6) are the bi-directional links or edge vector between pixels $p$ and $q$. The link is either horizontal, or vertical, or diagonal (relative to the position of $q$ in $p$ 's neighborhood). The dot product of $D(p)$ and $L(p, q)$ is positive, as noted in [6]. The direction feature cost is low when the gradient directions of the two pixels are similar to each other and the link between them.

Here, $f_{d i v}(p)$ is the divergence of unit gradient vector in the point of $p$. And $w_{d i v}$ is the weight of the term. Its function is to de-noise. There is not this term in the original model [6]. In order to de-noise we add this term to the model.

In (7), $\beta$ is small positive constant that prevent $\sqrt{I^{2}{ }_{x}+I^{2}{ }_{y}+\beta}$ to be zero. This term comes from the no-linear diffusion filter, first proposed by [4]. And it has been successful as a de-noise algorithm. $f_{d i v}(p)$ is sensitive to the oscillating such as noise but not penalize the step edges. So, in location of edge $f_{d i v}(p)$ is small, and in the location of noise $f_{d i v}(p)$ is big. The function of $f_{d i v}(p)$ will be demonstrated in the following of the paper.

\subsection{Searching for an Optimal Path}

As mentioned, dynamic programming can be formulated as a directed graph search for an optimal path. This paper utilizes an optimal graph search similar to that presented by Dijkstra [5]. Further, this technique builds on and extends previous boundary tracking methods in 4 important ways same as in [6], but the difference of 
our method is that we add the no-linear diffusion filter to the weighted map so that the search can resist the effect of noise. And all these characters can make MTF measurement easier.

The live-wire 2-D dynamic programming (DP) graph search algorithm is as follows: Figure 2.2 (a) is the initial local cost map with the seed point blacked. For simplicity of demonstration the local costs in this example are pixel based rather than link based and can be thought of as representing the gradient magnitude cost feature. Figure 2.2 (b) shows a portion of the cumulative cost and pointer map after expanding the seed point. Noticing that the diagonal local costs which have been scaled by Euclidean distance does not show in this figure. In fact we compute the diagonal local costs in our method, but for convenience we do not show them. This is demonstrated in Figure 2.2 (c) where several points have now been expanded, and the seed point and the next lowest cumulative cost point on the active list. In fact, the Euclidean weighting between the seed and diagonal points makes them more costly than nondiagonal paths. Figures 2.2 (d), (e), and (f) show the cumulative cost/direction pointer map at various stages of completion. Note how the algorithm produces a "wave-front" of active points emanating from the initial start point, which is called the seed point, and that the wave-front grows out faster where there are lower costs.

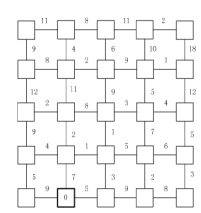

(a)

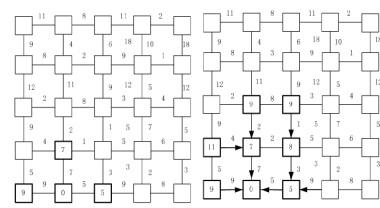

(b)

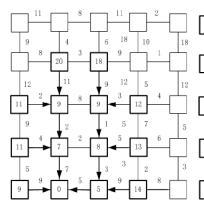

(d)

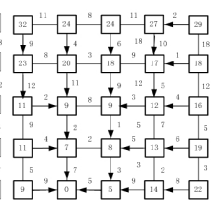

(e)

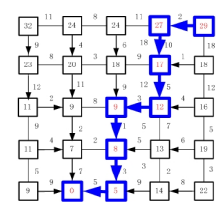

(f)

Fig. 2.2. (a)-(f) is the process of optimal path searching from one seed point to another. And the blacked points are the active points on the live paths.

And here, we have to say, the importance of the term of $f_{d i v}(p)$ is obvious. In the Figure 2.3, (a) is the edge extraction in the smooth ideal remote sense images. We can see that the result is satisfying. Even the edge is not straight or regular, the live-wire work well in (a). Following, (b) and (c) are the result or edge detection by live-wire model without the term $f_{\text {div }}(p)$, and they are badly affected by noise. In Figure 2.3(b) and (c), edge extraction is not optimal for us. Where the level of noise is high, the edge extraction is not very accurately. But, (d) is the result of live-wire edge extraction with the term $f_{d i v}(p)$. And we can see that result (d) is not affected by noise, and the boundary is on the right position for the functions of term $f_{d i v}(p)$. The term of $f_{d i v}(p)$ is sensitive to noise but almost does not change on the edge. So the term of no-linear diffusion filter operator $f_{d i v}(p)$ enhances the performance of the live-wire model very much. 


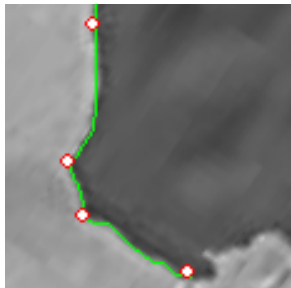

(a)

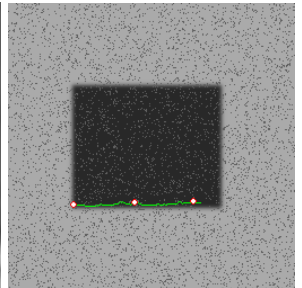

(b)

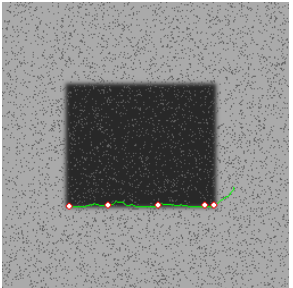

(c)

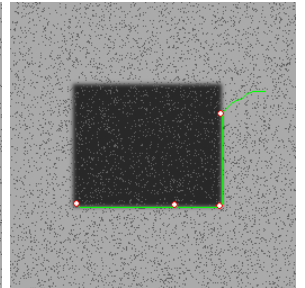

(d)

Fig. 2.3. (a) is the live-wire edge detecting of remote sensor image. (b) and (c) are the livewire edge detecting without $f_{d i v}(p)$ term and (d) is with the $f_{d i v}(p)$ term.

\section{MTF Measurement Based on Live-Wire}

Until now, we have acquired the knife-edges, and we must resample the profile that perpendicular to the edges same as [8]. On one edge we can acquire many profiles as Figure 3.1(b) shows. In order to check the accuracy of the algorithm, we use a known PSF to convolve the image of Figure 3.1 (a) and acquire the blur image of Figure 3.1 (b). The ideal MTF of the known PSF has been shown as figure 3.1 (g). Firstly, we

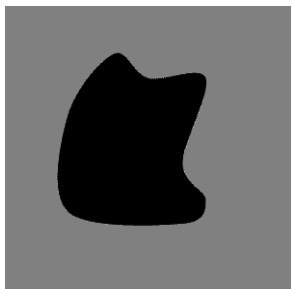

(a)

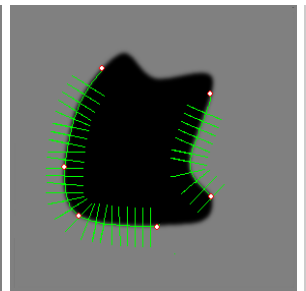

(b)

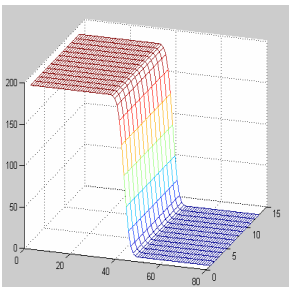

(c)

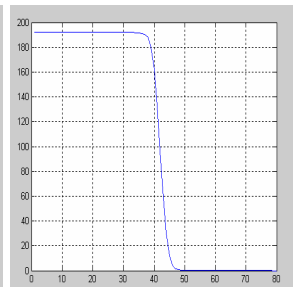

(d)

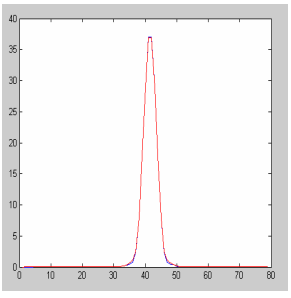

(e)

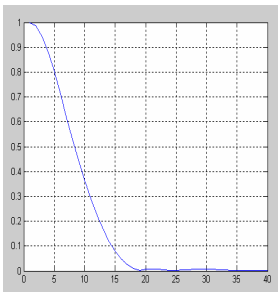

(f)

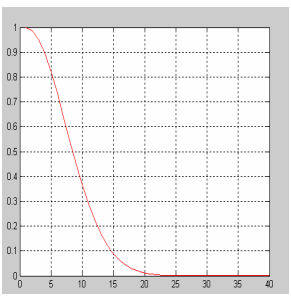

$(\mathrm{g})$

Fig. 3.1. MTF measurement in ideal situation

select the edge image of Figure 3.1 (b). Then, we use live-wire to search the optimal edge as knife-edge and compute the profiles that perpendicular to the edge as Figure 3.1 (b). Furthermore we use the minimum mean-square [8] value to compute the edge spread function as Figure 3.1 (d). Since the image Figure 3.1 (b) is ideal and simple, the ESF should be very accuracy. And the ESF was then differentiated to obtain the LSF as in the Figure 3.1(e). Then the LSF was Fourier-transformed and 
normalized to obtain the corresponding MTF Figure3.1 (f). The ideal MTF of (b) is Figure 3.1 (g). Comparing the Figure3.1 (f) with the Figure3.1 (g), we can find that the live-wire works very well and the result is very precise. There only is a little error in the MTF measurement, and it is out of the cut-off frequency. The amplitude frequency from 0 to 20 in (f) and (g) are almost the same, the error comes out only from 20 to 35 . The results do just verify the accuracy of the live-wire method. Figure3.2 (a) and (b) is the same target image of remote sensing, and the (c) and (d) are the MTF result of difference methods. In Figure 3.2 (c) and (d), the blue solid line is the MTF that is measured by method in [8]. And in Figure3.2 (c) the red solid line is the MTF that is measured by our algorithm. In Figure3.2 (d) the red solid line is the MTF that is measured by live-wire algorithm without improvement. The target image is irregular, but for the accurate edge extraction and more correct profile cutting, the result of red solid line that is based on our improvement is obviously more precise, see Figure 3.2 (c). Further more in Figure 3.2 (d), the red solid line is the MTF measured by original live-wire model that does not add the de-noise term of $f_{d i v}(p)$. We can see that for the affection of noise, the edges is not very accurate and it can affect the measurement of MTF in (d) and the error is also obvious. This illustrate the no-improved live-wire algorithm is not very suit for MTF measure.

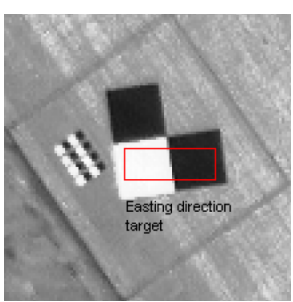

(a)

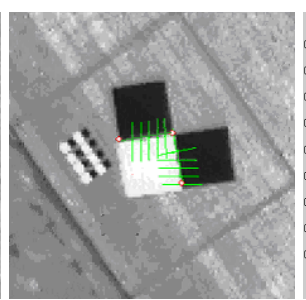

(b)

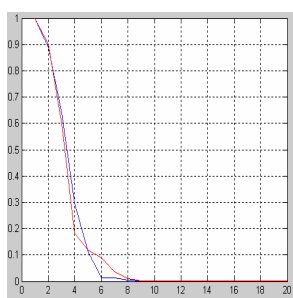

(c)

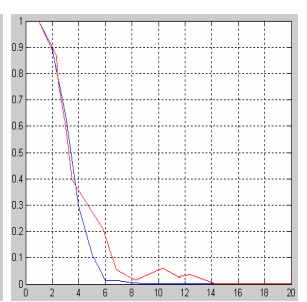

(d)

Fig. 3.2. MTF measurement based on difference methods

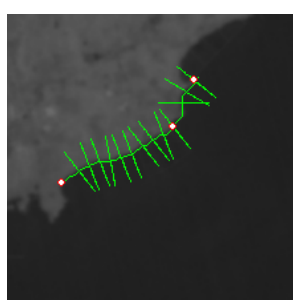

(a)

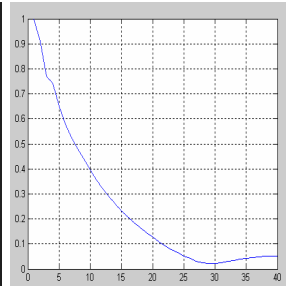

(b)

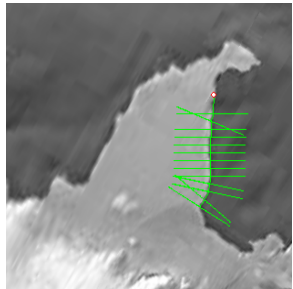

(c)

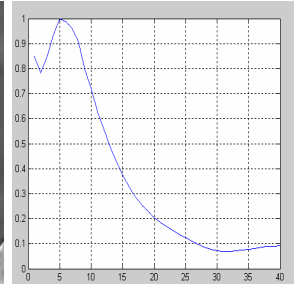

(d)

Fig. 3.3. MTF measurement of variety of remote sensing images

Figrure3.3 is MTF measurement used different images. In these figures we can see that, if the context in the image is complicated and the edge is not very straight we can also extract the edge accurately as Figure3.3 (a). And this success attribute to the advantages of the live-wire algorithm and the no-linear diffusion filter term added in 
live-wire model. At last we measure the MTF of the image of Figure3.3 (c) that comes from "Google earth". And live-wire can snap the edge easily and precisely on the complex image context. The profile that is perpendicular to the edge is cut and LSF is computed. Figure3.3 (d) is the MTF of Figure3.3 (c). Because the quality of the image is good, the result of MTF should be relatively ideal.

\section{Conclusions}

In this paper we propose an improved edge detection model based on live-wire algorithms to measure the MTF of remote sensing images. The no-linear filter term was added. And, these improvements make it more suitable for MTF measurement. It highly enhances the performance of the knife-edge method of MTF measurement and it makes the measurement more convenient and flexible. Then, we use the straight edge no more when we want to measure the MTF of the sensors directly. Furthermore, the influence of the noise is restrained after no-linear diffusion filter term is added to the weight map. The profiles that are perpendicular to edge can be simultaneously and accurately computed. So, all these advantages help us measure the MTF of the image more accurately in very complicated image context. The following work will focus on making use of the improved MTF parameters in de-convolution of remote sensing images.

\section{References}

1. W. A. Barrett and E. N. Mortensen. Interactive live-wire boundary extraction. Medical Image Analysis, 1(4):331-341, 1997.

2. M. Kass, A. Witkin, and D. Terzopoulos, "Snakes: Active Contour Models," in Proc. of the First Int. Conf. on Computer Vision, London, England, pp. 259-68, June 1987.

3. E. N. Mortensen and W. A. Barrett. Intelligent Scissors for Image Composition. Computer Graphics (SIGGRAPH '95), 191-198, 1995.

4. P. Perona, J. Malik, "Scale-space and edge detection using anisotropic diffusion", PAMI 12(7), pp. 629-639, 1990.

5. E. W. Dijkstra A Note On Two Problems in Connexion With Graphs Numerische Mathematik, 269-271, 1959.

6. A. X. Falcao, J. K. Udupa, and F. K. Miyazawa. An Ultra-Fast User-Steered Image Segmentation Paradigm: Live-wire on the Fly. IEEE Transactions on Medical Imaging, 19(1):55-61, 2000.

7. A. X. Falcao, J. K..Udupa, S. Samarasekera, and S. Sharma. User-Steered Image Segmentation Paradigms: Live-wire and Live Lane. Graphical Models and Image Processing, 60:233-260, 1998.

8. Taeyoung Choi, IKONOS Satellite on Orbit Modulation Transfer Function (MTF) Measurement using Edge and Pulse Method, thesis South Dakota State University.2002

9. E. N. Mortensen and W. A. Barrett. Interactive Segmentation with Intelligent Scissors. Graphical Models and Image Processing, 60(5):349-384, 1998. 